\title{
Plasmacytoid dendritic cells are scarcely represented in the human gut mucosa and are not recruited to the celiac lesion
}

\author{
M Ráki ${ }^{1,2}$, A-C Røberg Beitnes ${ }^{1}$, KEA Lundin ${ }^{1,3}$, J Jahnsen ${ }^{4}$, FL Jahnsen ${ }^{5}$ and LM Sollid ${ }^{1,6}$
}

Celiac disease (CD) is a chronic small intestinal inflammation precipitated by gluten ingestion. According to case reports, interferon (IFN) $\alpha$ administration may induce development of overt CD. Plasmacytoid dendritic cells (PDCs) were thought to be the source of IFN- $\alpha$ and promote a T helper type 1 response leading to lesion formation. Surprisingly and contradicting to earlier findings, PDCs were described as the main antigen-presenting cells (APCs) in human duodenal mucosa and particularly in CD. Here we show that when assessed by flow cytometry and in situ staining, PDCs represent $<1 \%$ of APCs in both normal duodenal mucosa and the celiac lesion. Low levels of IFN- $\alpha$ were detected in the celiac lesion assessed by western blot, reverse transcriptase (RT)-PCR, and immunohistochemistry. In four cell populations sorted from the celiac lesion (based on their expression of HLA-DR and CD45), we found that equally low levels of mRNA for IFN- $\alpha$ were distributed among these cell populations. Together, these results suggest that relatively small amount of IFN- $\alpha$, produced by a variety of cell types, is present in the celiac mucosa. IFN- $\lambda$, a type III IFN important in intestinal antiviral defense, was produced mainly by APCs, but its expression was not increased in the celiac lesion.

\section{INTRODUCTION}

Dendritic cells (DCs) are central in the immune system of the intestine for induction of both tolerogenic and effector immune responses. There are two main lineages of DCs, classical CD11c ${ }^{\text {hi }}$ myeloid and plasmacytoid DCs (PDCs). In addition, DCs derived from monocytes are also described in peripheral organs. DCs are important in both priming naive $\mathrm{T}$ cells in mesenteric lymph nodes and Peyer's patches and in the activation of effector $\mathrm{T}$ cells that reside in the intestinal lamina propria. Based mainly on studies in mice, the migratory $\mathrm{CD} 103^{+} \mathrm{CD} 1 \mathrm{c}^{+}$DCs are believed to be most important in priming of $\mathrm{T}$ cells in secondary lymphoid organs, ${ }^{1}$ while increasing evidence suggests that monocyte-derived DCs modulate local adaptive immune responses. ${ }^{2,3}$ PDCs are the major interferon (IFN)- $\alpha$-producing cells, which are necessary for defense against viruses, ${ }^{4}$ and probably also important in B-cell differentiation and antibody production. ${ }^{5}$
Reports on the features of PDCs in the gut are scarce. Most observations are from mice where PDCs are a minor population among the antigen-presenting cells (APCs) in the lamina propria. ${ }^{6-8}$ It was recently described that PDCs are able to home to the intestine of mice through CCR9 (C-C motif chemokine receptor 9), ${ }^{9}$ although it is difficult to assess whether they home to the lamina propria, as considerable numbers of isolated lymphoid follicles are present in the mouse small intestine. ${ }^{10}$ Further, in mice the CCR9 marker define immature, tolerogenic PDCs, which are also present as a resident population in resting lymphoid organs-notably not only in mesenteric lymph nodes-and which do not express high amounts of IFN- $\alpha .{ }^{11}$

In humans, PDCs are typically located in secondary lymphoid organs, but they can be recruited to various tumors and in pathological conditions of the skin and the airways. ${ }^{12-16}$

It was hypothesized that PDCs can be the source of IFN- $\alpha$ and promote Thelper type 1 response in celiac disease (CD) ${ }^{17} \mathrm{a}$

\footnotetext{
${ }^{1}$ Centre for Immune Regulation and Department of Immunology, Oslo University Hospital-Rikshospitalet, Oslo, Norway. ${ }^{2}$ Department of Pathology, Oslo University Hospital-Rikshospitalet, Oslo, Norway. ${ }^{3}$ Department of Medicine, Oslo University Hospital—Rikshospitalet, Oslo, Norway. ${ }^{4}$ Department of Gastroenterology, Oslo University Hospital—Aker, Oslo, Norway. ${ }^{5}$ Centre for Immune Regulation and Department of Pathology, Oslo University Hospital—Rikshospitalet and University of Oslo, Oslo,

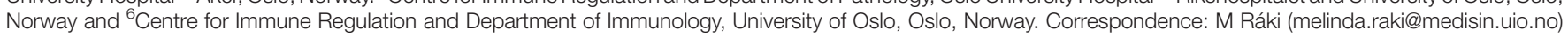


chronic small intestinal inflammation that is precipitated by wheat gluten ingestion and driven by activation of gluten-reactive CD4 T cells of the intestine. ${ }^{18} \mathrm{~A}$ link between IFN $-\alpha$ and $C D$ is supported by increased IFN- $\alpha$ expression in the celiac lesion compared with normal intestinal mucosa ${ }^{19}$ and the clinical observation that IFN- $\alpha$ administration may induce development of overt CD. ${ }^{19-25} \mathrm{Di}$ Sabatino et al. ${ }^{17}$ found that PDCs are the main APC population in the small intestine and that they are responsible for most of the increase in DC numbers observed in the celiac lesion. Our initial observations ${ }^{26}$ suggested that PDCs are scarce in the small intestine. In this study, we examined the presence of PDCs in the celiac lesion and the presence and source of IFN- $\alpha$.

\section{RESULTS}

\section{Few PDCs are present in the duodenal mucosa}

DCs in blood are defined by HLA-DR expression and lack of T-cell, B-cell, natural killer cell and monocyte markers, socalled lineage negative cells. PDCs in blood express high levels of CD123, and also CD45RA, BDCA2, BDCA4, and CD62L. In order to examine the proportion of PDCs in the duodenal mucosa, single-cell suspensions were prepared by digesting duodenal biopsies with collagenase followed by staining for lineage markers (CD3, CD14, CD19, CD20, CD56, and in some experiments, also CD34), HLA-DR, CD123, and CD45RA. CD $123{ }^{\text {hi } C D 45 R A}{ }^{+}$cells were barely detectable in the lin $^{-}$HLA-DR $^{+}$population (Figure 1a,b), except in one treated $\mathrm{CD}$ patient in whom about $6 \%$ of $\mathrm{Lin}^{-} \mathrm{DR}^{+}$cells
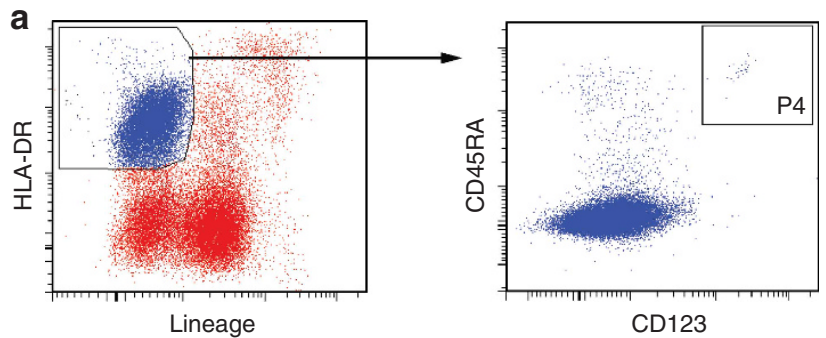

b
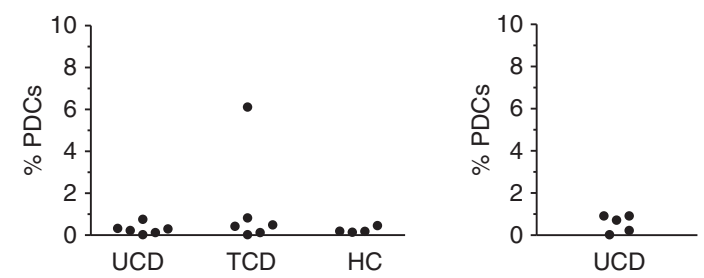

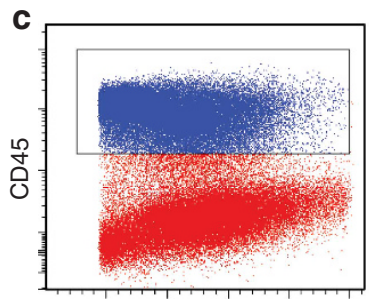

FSC

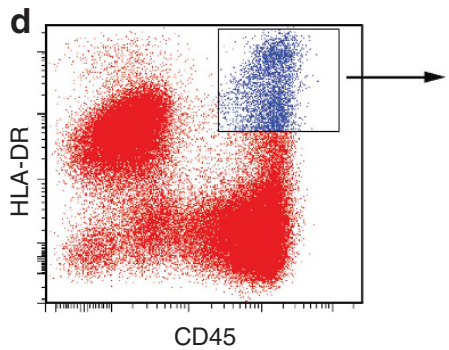

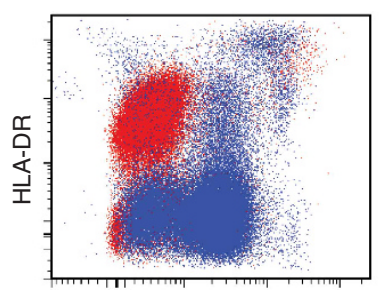

Lineage

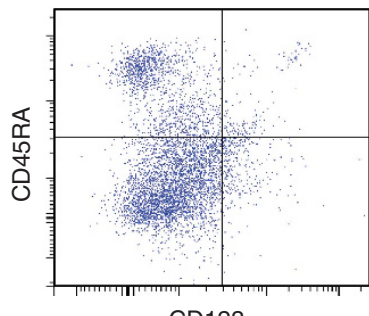

CD123

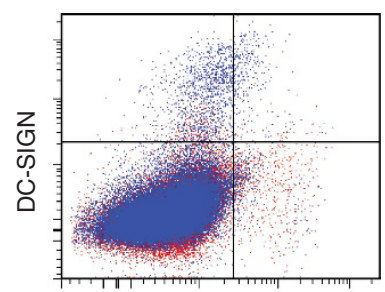

CD123

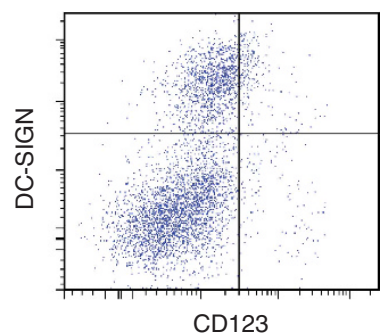

Figure 1 Plasmacytoid dendritic cells (PDCs) are scarce in the human duodenal mucosa. Biopsy specimens from duodenal mucosa of celiac disease patients and controls were digested with collagenase after removal of the epithelial layer by incubation with EDTA. The obtained single-cell suspensions were then stained for various markers. (a) CD123 and CD45RA expression on lin ${ }^{-}$HLA-DR $^{+}$cells. Cells in P4 are considered as PDCs. (b) Frequency of $\mathrm{CD}_{123}{ }^{+} \mathrm{CD}_{45 \mathrm{RA}}{ }^{+}$PDCs among lin ${ }^{-} \mathrm{HLA}^{-D R^{+}}$cells (left panel) and CD45 ${ }^{+} \mathrm{HLA}^{-D R^{+}}$APCs (right panel) in untreated (UCD) and treated celiac disease (TCD) patients and healthy controls (HC). (c) Most lin ${ }^{-}$HLA-DR $^{+}$cells are not leukocytes. CD45-expressing cells are shown in blue in all the three plots. Some DC-SIGN ${ }^{+}$cells express low levels of CD123. Some non-leukocytes (endothelial cells) express high levels of CD123. (d) CD123 and CD45RA expression levels on HLA-DR ${ }^{+} \mathrm{CD}_{4}{ }^{+}$antigen-presenting cells. Some DC-SIGN ${ }^{+}$cells express low levels of CD123. DC-SIGN, dendritic cell-specific intercellular adhesion molecule-3-grabbing non-integrin; FSC, forward scatter. 
expressed CD123 and CD45RA, which we believe could have originated from a lymphoid aggregate (Figure 1b). In tissue digests, however, many cell types are not covered by the lineage markers. Some of these cells also express MHC (major histocompatibility complex) class II molecules, such as epithelial and endothelial cells, of which the latter are also positive for CD123. Indeed, most lin ${ }^{-}$HLA-DR ${ }^{+}$cells in the tissue digests were not leukocytes (Figure 1c). Among APCs defined as viable (PI negative) cells expressing HLA-DR and CD45 (Figure 1d), $<1 \%$ were PDCs (Figure 1b). Of note, most $\mathrm{CD} 123^{\text {hi }}$ cells were CD45-negative (Figure 1c). As expected, additional co-staining experiments showed that these were endothelial cells (not shown). Low expression of CD123 was found on some macrophages expressing DC-SIGN (dendritic cell-specific intercellular adhesion molecule-3-grabbing nonintegrin; Figure 1c,d).

Expression of Toll-like receptor 9 (TLR9) by PDCs was used to argue for abundance of PDCs in the duodenal mucosa. ${ }^{17} \mathrm{We}$ thus looked at TLR9 expression by flow cytometry and found no abundant population of PDC-like cells expressing TLR9. Rather, TLR 9 was expressed at high levels by $\mathrm{CD} 27^{+}$cells being B cells and plasma cells (Supplementary Figure S1A) and at lower levels by a variety of other cell types, including CD45 ${ }^{-}$ cells (mainly epithelial cells) and $\mathrm{CD}^{+} 4^{+}$macrophages (Supplementary Figures S1B,1C).

Detection of PDCs is not altered by collagenase digestion To ascertain whether the scarcity of PDCs could relate to cleavage of $\mathrm{CD} 123$ by collagenase during tissue digestion, peripheral blood mononuclear cells were incubated with collagenase and, following CD45-labeling, mixed with unlabeled cells from duodenal mucosa in order to assess the effect of enzymatic digestion. The mixed cell suspension was then stained for lineage markers, HLA-DR and CD123, to identify PDCs. Cells that were derived from blood could be distinguished by positive CD45 Pacific Blue staining, while duodenal cells appeared CD45 negative, as the staining was done before mixing of the populations. PDCs from blood were readily detected being $\mathrm{CD}_{4} 5^{+}$and expressing high levels of CD123, which demonstrated that the collagenase treatment did not affect the expression of this receptor. Some very few PDCs from the duodenal digest were also identified being $C D 45^{\text {neg }}$ $\mathrm{CD}_{123}{ }^{+}$(Figure 2).

\section{Density of PDCs is unaltered in the celiac lesion}

We did not find a difference in the percentage of PDCs in the duodenum of control individuals and $\mathrm{CD}$ patients by flow cytometry (Figure 1b). To establish PDC density, we enumerated PDCs in sections of duodenal mucosa from untreated CD patients and control patients with normal histology. Sections from frozen biopsies were stained for CD123 and either CD45RA or von Willebrand factor. PDCs were defined as cells co-expressing CD45RA and CD123, as previously described. ${ }^{14}$ Numerous cells expressed CD123, but the morphology of these resembled vessels, what was corroborated by staining of von Willebrand factor. Consistent with the results from flow cytometry, very few PDCs were found by in situ staining (Figure 3). The density of PDCs was unaltered in untreated $\mathrm{CD}$ (median 12 cells per $\mathrm{mm}^{2}$; Figure 4) and corresponded to $<1 \%$ of HLA-DQ ${ }^{+}$APCs, which we found to be about 1500 cells per $\mathrm{mm}^{2}$ in our earlier studies. ${ }^{26,27}$

\section{Detectable IFN- $\alpha$ by western blot in the celiac lesion}

Next we determined whether IFN- $\alpha$ expression is detectable in the celiac lesion. Lysates were made from whole biopsies, and western blot was performed with various amounts of human recombinant IFN $\alpha$ as positive control. IFN $-\alpha$ protein was found in seven of nine untreated CD patients but not in five control subjects (Figure 5). Notably, we needed to load high amounts of protein, $150-200 \mu \mathrm{g}$ per lane from whole-biopsy lysates to be able to detect IFN- $\alpha$, indicating that there is probably little IFN $-\alpha$ in the celiac lesion.

To examine the cellular source of IFN- $\alpha$ in the celiac lesion, we first stained single-cell suspensions from three untreated celiac patients for IFN- $\alpha$ for evaluation by flow cytometry. IFN- $\alpha$ staining was negative in all the three CD patients (data not shown), probably due to high background and low concentration of IFN- $\alpha$ in individual cells. Of note, IFN- $\alpha$ was readily detected in PDCs of PBMC that was stimulated with CpG for $6 \mathrm{~h}$ (not shown).

We also made single-cell suspensions that were stained for HLA-DR and CD45 and four populations were sorted by flow cytometry (Supplementary Figure S2A). Cells were lysed, and

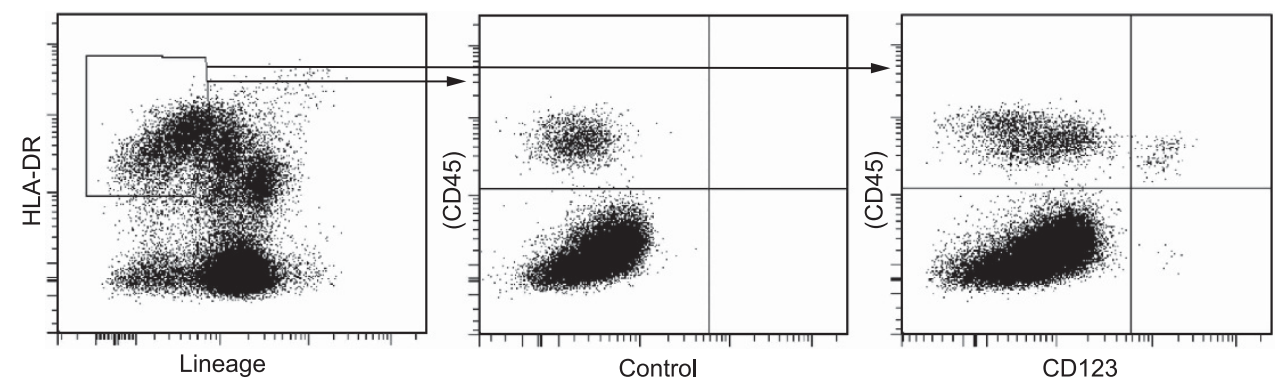

Figure 2 Collagenase digestion does not interfere with detection of plasmacytoid dendritic cells (PDCs). Peripheral blood mononuclear cells (PBMC) were digested with collagenase and stained for CD45 Pacific Blue. Subsequently, CD45-labeled PBMC were mixed with single-cell suspension obtained from small intestinal biopsies from the same patient. Mixed cells were then stained for lineage markers, HLA-DR and CD123. CD123 expression was assessed in lin $^{-}$HLA-DR $^{+}$cells. Most CD123 ${ }^{\text {hi }}$ PDCs were among the CD45-labeled PBMC. 

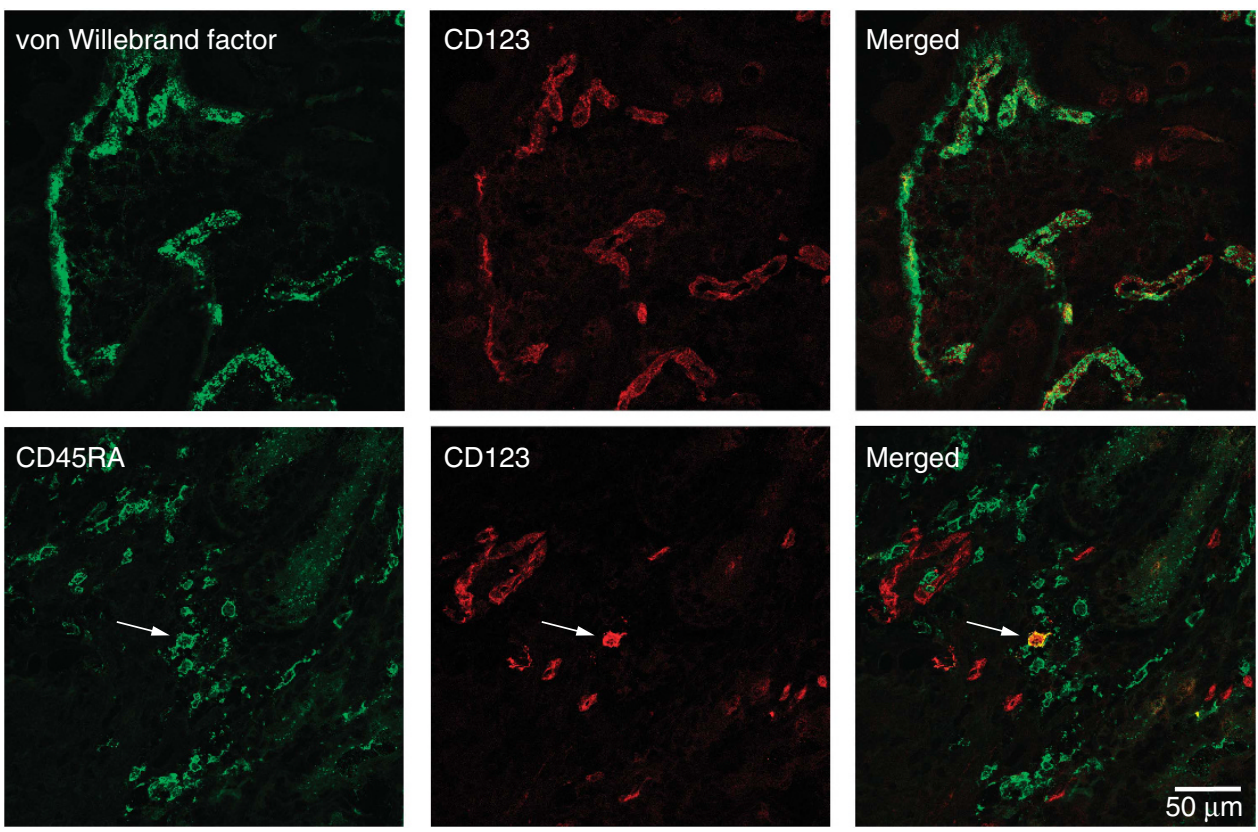

Figure 3 Most CD123 $3^{\text {hi }}$ cells are capillary endothelium cells in sections from duodenal mucosa. Cryosections of duodenal mucosa were stained for CD123 (red) and either von Willebrand factor (green, upper panels) or CD45RA (green, lower panels). Note that most von Willebrand factor + cells express CD123, whereas only one CD45RA + CD123 + cell was found (arrow). Original magnification $\times 600$.

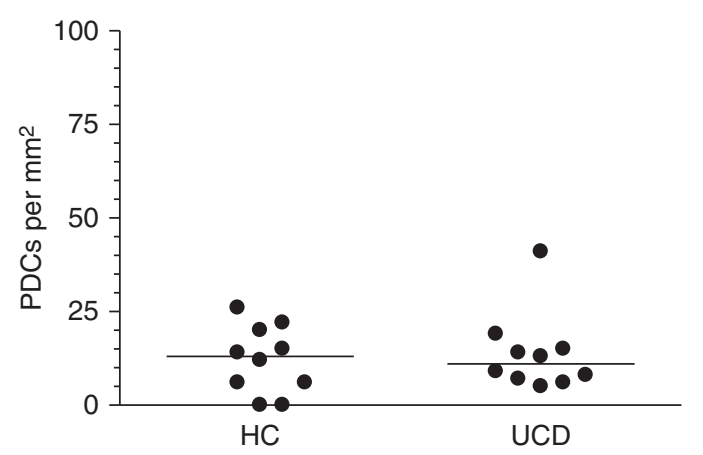

Figure 4 The density of $\mathrm{CD} 45 \mathrm{RA}{ }^{+} \mathrm{CD}_{123^{+}}$plasmacytoid dendritic cells (PDCs) does not increase in the celiac lesion. Cryosections from duodenal mucosa were stained for CD123 and CD45RA. Density of PDCs was evaluated by superimposing a grid on sections and the number of CD45RA ${ }^{+} \mathrm{CD}_{123^{+}}$cells was calculated per $\mathrm{mm}^{2}$ lamina propria. Horizontal lines indicate median. HC, healthy controls; UCD, untreated celiac disease.

IFN- $\alpha$ content in the different cell populations was assessed by western blotting. Due to low signals, we were not able to reliably identify which cell populations contributed to the IFN- $\alpha$ protein production (not shown).

\section{Myxovirus resistance gene A (MxA) expression is increased in CD}

Finally, we examined the expression of MxA, a widely used surrogate marker for type I IFNs, in samples from the duodenal mucosa and skin lesions from discoid lupus erythematosus. ${ }^{12}$ MxA expression was graded from 0 to 5 based on staining within the epithelium as described in the Methods section. MxA expression was increased in the epithelial cells in the celiac

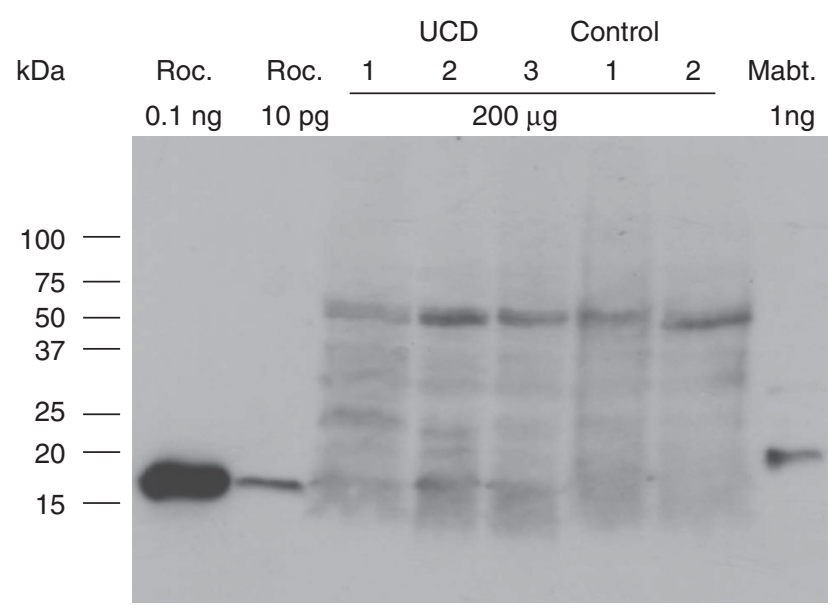

Figure 5 Interferon (IFN)- $\alpha$ protein is detectable in lysates from the duodenal mucosa of most celiac disease (CD) patients by western blot at $18-20 \mathrm{kDa}$. In all, 1-3 biopsy specimens were lysed and cleared by centrifugation. Lysates containing $200 \mu \mathrm{g}$ total protein were loaded onto each lane of a $15 \%$ sodium dodecyl sulfate gel. IFN- $\alpha$ was detected with mouse monoclonal antibodies after transferring the proteins onto a nitrocellulose membrane. Molecular weight markers indicated on the left. Lanes 1-2: positive control, 100 and $10 \mathrm{pg}$ recombinant IFN- $\alpha 2 a$

(Roceron; Roc.); lanes 3-5: lysates from three untreated celiac disease (UCD) patients; lanes 6-7: lysates from two control patients; and lane 8: positive control containing $1 \mathrm{ng}$ of a mix of various IFN- $\alpha$ subtypes (from Mabtech; Mabt.). Of note, there is an unspecific band at about $60 \mathrm{kDa}$ in all lysates.

lesion compared with normal duodenal mucosa (Figure 6), indicating type I IFN stimulus. However, in skin lesions from lupus erythematosus, $\mathrm{MxA}$ expression was considerably higher than in the celiac lesion. In addition, in contrast to the lamina propria in the celiac lesion, we found remarkably high MxA 
expression in the dermis of lupus skin probably corresponding to accumulating PDCs. ${ }^{12}$

Indeed, similar amount of IFN- $\alpha$ mRNA was found in four cell populations sorted according to CD45 and HLA-DR expression, supporting that there are several cell types producing this cytokine in the small intestinal lamina propria (Supplementary Figure S2), although IFN- $\alpha 5$ mRNA expression was significantly increased in $\mathrm{CD}_{4} 5^{+} \mathrm{DR}^{-}$ leukocytes, consisting mainly of $\mathrm{T}$ cells, plasma cells, and granulocytes and in $\mathrm{CD}_{4} 5^{-} \mathrm{DR}^{-}$cells, representing among others fibroblasts, goblet cells, and smooth muscle cells, from the celiac lesion. Moreover, 500-1000 times less IFN- $\alpha$ mRNA was found in biopsies compared with similar amounts of PBMC (which contained about 40-80 PDCs) that was stimulated with CpG for $4 \mathrm{~h}$ (Supplementary Figure S2).

\section{IFN- $\lambda$ expression is not increased in the celiac lesion}

MxA expression is also induced by type III IFNs that were recently shown to have an important role in intestinal rotavirus infection in mice. ${ }^{28}$ Frequent rotavirus infections are suspected to be associated with increased risk of developing $\mathrm{CD},{ }^{29}$ therefore we examined IFN- $\lambda$ expression. Measured by enzyme-linked immunosorbent assay (ELISA), similar concentrations of IFN- $\lambda$ were found in duodenal biopsies from CD patients and controls (Figure 7). When assessed by RT-PCR, we found that IFN- $\lambda$ mRNA was mainly expressed by APCs in the celiac lesion but with higher expression of IFN- $\lambda 1$ in controls than CD patients (Supplementary Figure S2).

\section{DISCUSSION}

Here we show that very few PDCs are present in the small intestinal mucosa, and the density of these cells does not increase in CD. IFN- $\alpha$ protein at moderate levels is detectable by western blotting in most patients with untreated CD but not in controls. Several cell types in the CD lesion produce IFN- $\alpha$ as assessed by mRNA expression.
PDCs were $\operatorname{lin}^{-} \mathrm{HLA}-\mathrm{DR}{ }^{+} \mathrm{CD}_{4}{ }^{+} \mathrm{CD} 123^{+} \mathrm{CD}_{45 \mathrm{RA}}{ }^{+}$ cells as expected and similar numbers were found with various gating strategies, indicating that we did not overlook a part of the population. Di Sabatino et al. ${ }^{17}$ argued that most APCs in the lamina propria are PDCs, what is in conflict with our comprehensive analysis. Based on our results, we suggest an alternative interpretation of their observations. We believe that the cells expressing high levels of CD123 in their immunohistochemical analysis were endothelial cells, while the CD123positive cells in their flow cytometry experiments presumably represented macrophages or myeloid DCs, which are known to express CD123 at low levels. ${ }^{30,31}$ In immunohistochemistry, these cells may express CD123 at too low levels for efficient detection. Further, we found that macrophages or myeloid DCs dimly expressed TLR9, which could explain the findings of Di Sabatino et al. ${ }^{17}$ of an abundant TLR9-expressing population of cells.

PDCs are mainly located in lymphoid tissues, although in some cases they can be found in inflamed tissues. ${ }^{12,14,16}$ Several reports have shown recruitment of PDCs to skin ${ }^{12,13,16}$ and airways, ${ }^{14,15}$ while there is little evidence for recruitment to

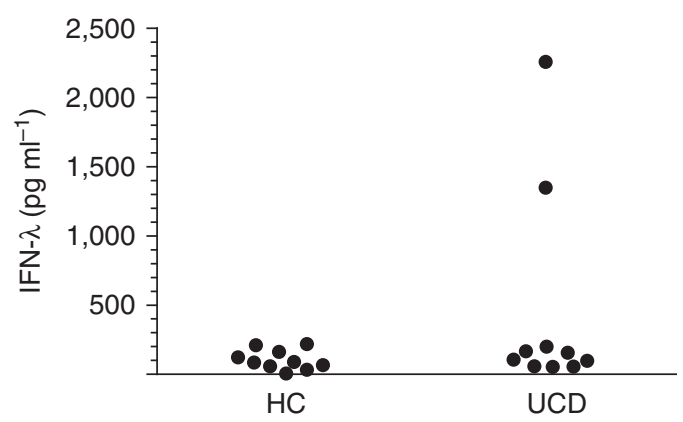

Figure 7 Interferon (IFN)- $\lambda$ expression is similar in duodenal biopsies from healthy controls (HC) and untreated celiac disease patients (UCD). IFN- $\lambda$ concentration was measured in duplicates in biopsy lysates containing $50 \mu \mathrm{g}$ total protein.
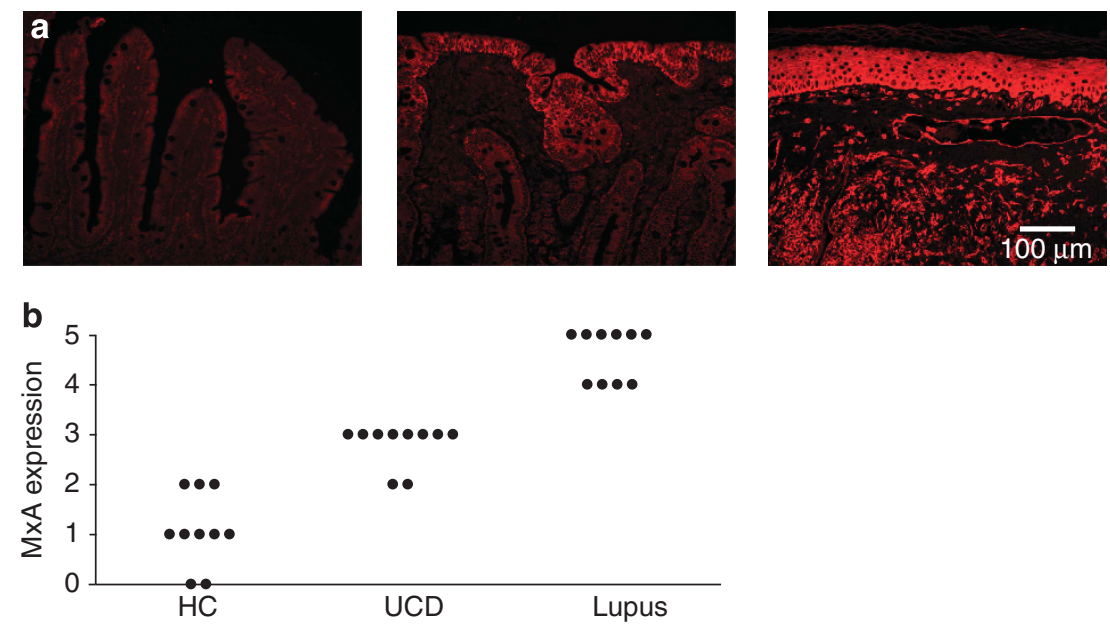

Figure 6 Myxovirus resistance gene A (MxA) expression is increased in the celiac lesion and even more in skin lesions from discoid lupus erythematosus. (a) Immunofluorescence staining for MxA (red) in formalin-fixed and paraffin-embedded section of duodenal mucosa from a normal healthy control (HC; left panel), an untreated CD patient (UCD; middle panel), and skin from a patient with lupus (right panel). Original magnification $\times 400$. (b) Epithelial MxA expression in $10 \mathrm{UCD}, 10 \mathrm{HC}$ and 10 patients with lupus, graded $0-5$ as described in the Methods section. 
intestinal lamina propria in humans. Indeed, our finding of very few PDCs in the small intestinal mucosa is in agreement with another study. ${ }^{32}$ We conclude that PDCs are scarce in the small intestine, and they are not recruited into the celiac lesion.

Given our failure to detect accumulation of PDCs in the celiac lesion, an imminent question is whether IFN- $\alpha$ is produced in the celiac mucosa, and if so, by which cells. Although we were unable to detect IFN- $\alpha$ by intracellular flow cytometry, we were able to detect IFN- $\alpha$ in lysates of duodenal biopsies of untreated $\mathrm{CD}$ patients in western blot. This confirms earlier findings. ${ }^{19}$ Notably, however, the total protein loaded per lane by us $(150-200 \mu \mathrm{g})$ and in the previous report $(300 \mu \mathrm{g})$ is considerably more than what is usually used $(10-40 \mu \mathrm{g})^{33,34}$ in western blot, suggesting that little IFN- $\alpha$ protein is present in the celiac lesion.

To determine the source of IFN- $\alpha$, we proceeded with RT-PCR. IFN- $\alpha$ comprises multiple subtypes: 14 IFN- $\alpha$ genes give rise to 12 distinct human IFN- $\alpha$ proteins. ${ }^{35}$ Although upon viral infection many different cells can produce type I IFNs, PDCs are the main systemic source of this cytokine and secrete 200-1000 times more than other cell types. ${ }^{36}$ Importantly, most cells usually produce several subtypes of IFN- $\alpha$. We chose to examine five subtypes. We found that the examined cell populations from duodenal biopsies expressed mRNA for several subtypes of IFN- $\alpha$ but in considerably lower amounts than blood-derived PDCs that were stimulated with CpG. Together, these results suggest that relatively small amount of IFN- $\alpha$, produced by a variety of cell types, is present in the celiac mucosa. This notion is corroborated by MxA staining. MxA was expressed much weaker both in celiac enterocytes and in the lamina propria than in the epidermis and underlying dermis from lupus erythematosus lesions, the model for pathogenic relevance of type I IFN in autoimmunity.

Comparing patients with $\mathrm{CD}$ with controls, IFN- $\alpha 5$ mRNA was significantly more abundant in $\mathrm{CD}_{4} 5^{+} \mathrm{HLA}^{-\mathrm{DR}^{-}}$leukocytes and $\mathrm{CD}^{-} 5^{-}$HLA-DR ${ }^{-}$cells from CD patients. In addition, we observed a tendency for increased expression of several IFN- $\alpha$ subtypes in leukocytes and in the CD $45^{-}$HLA$\mathrm{DR}^{-}$population, although it did not reach significance, probably due to the limited number of patients tested. Little is known about the role of different subtypes of IFN- $\alpha$, although there are studies that suggest different kinetics and efficiency in various infections and cell types. ${ }^{35}$ For instance, IFN- $\alpha 5$ was shown to induce stronger Stat1 (signal transducer and activator of transcription factor) signaling and higher expression of the antiviral gene $2^{\prime} 5^{\prime}$ oligoadenylate synthetase in liver cells than IFN- $\alpha 2{ }^{37}$

We found a slight upregulation in IFN- $\alpha$ detected by western blot and RT-PCR in biopsies from CD patients but mainly in leukocytes and stromal cells, whereas downstream effect, as assessed by MxA staining, was seen mostly in the epithelium. This might be explained by a paracrine effect of IFN- $\alpha$. We altogether examined $27 \mathrm{UCD}$ patients for IFN- $\alpha$ or MxA expression with various assays. Even though a limited number of patients were examined with each assay, the different assays gave consistent results, strongly suggesting that expression heterogeneity and skewed representation of patients have not misled our conclusion about the IFN- $\alpha$ expression in CD.

Virus infections, especially frequent rotavirus infections, have been linked with CD, and IFN- $\lambda$ has been shown to have critical role in intestinal antiviral defense in mice. Interestingly, we observed that in the duodenal mucosa, IFN- $\lambda$ was almost exclusively produced by APCs. However, mRNA levels of IFN$\lambda 1$ were higher in controls than in CD patients. In ELISA, 2 out of $10 \mathrm{CD}$ patients had considerably increased IFN- $\lambda$ concentration, indicating that there may be differences among subgroups of patients and viral infections may have a role in some patients. In our material, the two patients with highest IFN $-\lambda$ concentrations had abdominal pain for more than a year but without a history of preceding gastrointestinal infection. One of the other patients had initial symptoms with vomiting and diarrhea a half year before diagnosis that were considered as gastrointestinal infection, but which resolved by the time of the diagnosis. Stool culture for bacteria was negative and blood for virus serology was unfortunately not taken. This latter patient had normal/low IFN- $\lambda$ level, but it is possible that initial high IFN- $\lambda$ levels decrease with time. An association between $\mathrm{CD}$ and virus infections is difficult to prove without prospective studies designed specifically for this purpose because of the long time between appearance of symptoms and the diagnosis of CD.

A variety of autoimmune phenomena have been reported after IFN- $\alpha$ therapy. In contrast to thyroid dysfunction, ${ }^{38}$ there are only a few case reports on $\mathrm{CD}$, which may indicate a relatively weak association. We are aware of case reports on nine patients who were diagnosed with $\mathrm{CD}$ during IFN- $\alpha$ therapy. For many cases, it is hard to conclude whether IFN- $\alpha$ therapy initiated the disease or just aggravated the disease and symptoms as pretreatment serum samples were either not available ${ }^{19,23-25}$ or they were scored positive for endomysial antibodies. ${ }^{20-23}$ IFN- $\alpha$ may aggravate the disease, for instance, by enhancing maturation and antigen presentation by DCs and prolonging survival of plasma cells and thus increasing antibody production against TG2.

Taken together, our study demonstrates that PDCs are rare in the intestinal mucosa of normal subjects as well as in patients with $\mathrm{CD}$. This cell type is thus not likely a key player in the pathogenesis of $\mathrm{CD}$. Although there is moderately increased expression of IFN- $\alpha$ and Mxa in the active celiac lesion, our results do not indicate an overly increased mucosal production of type I or type III IFNs in this enteropathy.

\section{METHODS}

Subjects. Multiple duodenal biopsy specimens were obtained from patients with untreated $\mathrm{CD}(n=36$, mean age 37.3 years; range $20-72$ years; 25 women) or treated CD $(n=10$, mean age 45.1 years, range 28-67 years; 7 women) and patients with normal histology ( $n=26$, mean age 43.6 years, range $20-85$ years; 18 women) who were examined with gastroendoscopy as part of the routine diagnostic workup. CD was diagnosed based on clinical symptoms, serology, and typical histological changes. Control patients had been referred to gastroendoscopy for abdominal pain, dyspepsia, diarrhea, or weightloss, and histological examination showed normal duodenal mucosa 
(Marsh 0). The study complies with the declaration of Helsinki and was approved by the regional committee for medical research ethics. All patients gave written informed consent to the study. To assess MxA expression in skin, archive biopsies were used from a material published in 2001. ${ }^{12}$

Multicolor flow cytometry. For detection of cell surface markers in single-cell suspensions, duodenal biopsies were incubated with $2 \mathrm{~mm}$ EDTA for $60 \mathrm{~min}$ to remove epithelial cells and intraepithelial leukocytes, and the remaining material was digested with $1 \mathrm{mg} \mathrm{ml}^{-1}$ blend collagenase (Sigma, C-8051, St Louis, MO) for 60 min at $37^{\circ} \mathrm{C}$. Cells were dispersed and stained with directly labeled antibodies for 30 min on ice in the dark followed by analysis on FACS Calibur or LSRII instruments (BD Biosciences, San Diego, CA). Dead cells were excluded by adding propidium iodide. For intracellular staining and sorting of cell populations for RT-PCR, biopsies were only incubated with collagenase to include epithelial cells. For intracellular staining (TLR9, clone eB72-1665 and IFN- $\alpha$, clone 7N4-1, both from BD bioscience), cells were resuspended in $1 \%$ paraformaldehyde after staining of surface antigens and stored overnight at $4{ }^{\circ} \mathrm{C}$. Samples were then permeabilized with $0.2 \%$ saponin $/ 3 \%$ fetal calf serum/phosphatebuffered saline for $30 \mathrm{~min}$ followed by staining with control antibody, anti-TLR9, or anti-IFN- $\alpha$ antibody in the same medium.

The following antibodies were used: anti-CD45-FITC (fluorescein isothiocyanate), -PE (phycoerythrin), -APC (allophycocyanin), (all clone HI30), anti-CD45-eFluor450 (clone HI30), anti-HLADR-eFluor450 (clone L243), and anti-CD27-PE-Cy7 (clone LG7F9) from eBioscience, San Diego, CA; anti-CD14-APC-Cy7 (clone HCD14) from Biolegend, San Diego, CA; anti-CD123-PE and APC (clone AC145) from Miltenyi Biotec, Bergisch Gladbach, Germany; anti-CD11c-PE (clone S-HCL-3), anti-DC-SIGN-PE and -APC (both clone DCN46), anti-CD11c-APC and -V450 (both clone B-Ly6), lineage cocktail-FITC (clones: SK7, 3G8, SJ25C1, L27, M $\varphi$ P9, NCAM 16.2) 1/20, anti-HLA-DR-PE-Cy7 (clone L243), and antiCD45RA-APC (clone HI100) from BD Biosciences. Isotype controls for FITC, PE, and APC were from BD Biosciences, for Pacific Blue and APC-Cy7 from Biolegend and ,for PE-Cy7 from eBioscience.

Immunohistochemistry. For enumeration of PDCs, cryosections were used. Duodenal biopsy specimens were oriented on thin slices of carrot, embedded in Tissue Tek optimal cutting temperature compound, snap-frozen "bed-side" in liquid nitrogen, and stored at $-70^{\circ} \mathrm{C}$. Cryosections were cut in series at $4 \mu \mathrm{m}$, dried in room temperature (RT) over night, fixed with acetone for $10 \mathrm{~min}$, dried for $15 \mathrm{~min}$, wrapped in aluminum foil, and stored at $-20^{\circ} \mathrm{C}$ until use. The cryosections were incubated with combinations of mouse monoclonal antibodies (mAbs) to human CD123 (clone 7G3, BD Pharmingen) or CD45RA (clone L48, BD Pharmingen) and a rabbit polyclonal antibody specific for von Willebrand factor (Dako, Glostrup, Denmark) for $1 \mathrm{~h}$ at RT. The sections were then incubated with biotinylated goat anti-mouse immunoglobulin G2a (IgG2a; Southern Biotech, Birmingham, AL) for $1.5 \mathrm{~h}$, followed by a combination of Cy2-labeled streptavidin (Amersham Biosciences, Buckinghamshire, UK) and Cy3-labeled goat anti-mouse IgG1 (Southern Biotech) or Alexa 488labeled goat anti-rabbit IgG (Molecular probes, Eugene, OR) for $30 \mathrm{~min}$. All incubations were followed by gentle wash for $2 \mathrm{~min}$ in phosphate-buffered saline.

In situ IFN- $\alpha / \beta$ production was evaluated by immunofluorescence staining for MxA, an IFN- $\alpha / \beta$ inducible intracellular protein well established as a surrogate marker for local IFN- $\alpha / \beta$ production. ${ }^{39}$ Formalin-fixed and paraffin-embedded duodenal biopsies were cut in $4 \mu \mathrm{m}$ sections and dewaxed. The sections were subjected to heat-induced epitope retrieval in citrate buffer $(\mathrm{pH}=6,1)$ by boiling at $100{ }^{\circ} \mathrm{C}$ in a water bath for $20 \mathrm{~min}$ and subsequent cooling to RT for $20 \mathrm{~min}$. The sections were then incubated overnight at $4{ }^{\circ} \mathrm{C}$ with $\mathrm{mAb}$ to human $\mathrm{MxA}$ (clone $\mathrm{M} 143,{ }^{40}$ kind gift from $\mathrm{O}$. Haller, Freiburg, Germany), followed by incubation with Cy3-labeled goat anti-mouse IgG2a (Southern Biotech) for $2 \mathrm{~h}$ at RT. Irrelevant isotype- and concentration-matched primary mAb was used as negative control.

Fluorescence microscopy. Cryosections and formalin-fixed sections were examined at $\times 400$ magnification in a fluorescent microscope (Nikon Eclipse 80i, Nikon, Melville, NY). The density of PDCs was determined by counting all $\mathrm{CD} 123{ }^{+} \mathrm{CD} 45 \mathrm{RA}^{+}$cells in the lamina propria to a depth of $\sim 0.5 \mathrm{~mm}$ from the basolateral side of the surface epithelium by superimposing a grid $(10 \times 10$ lines; $0.242 \times 0.242 \mathrm{~mm})$ parallel to the muscularis mucosa. On average, 10 grids were examined for every section.

MxA expression was graded from 0 to 5 based on staining within the epithelium as follows: Grade $0=<10 \%$ of the epithelium is weakly positive; Grade $1=10-50 \%$ of the epithelium is weakly positive; Grade $2=>50 \%$ of the epithelium is weakly positive; Grade $3=100 \%$ of the epithelium is weakly positive; Grade $4=100 \%$ of the epithelium is strongly positive; and Grade $5=$ very strong positivity in $100 \%$ of the epithelium.

Western blot. For western blot, bedside biopsies were frozen in liquid $\mathrm{N}_{2}$, and stored at $-70^{\circ} \mathrm{C}$. In all, $1-3$ biopsies were incubated in $100-150 \mu \mathrm{l}$ lysis buffer containing $50 \mathrm{~mm}$ Tris- $\mathrm{HCl}, 150 \mathrm{~mm} \mathrm{NaCl}$, $1 \mathrm{~mm}$ EDTA, $1 \%$ NP-40, 0,1\% sodium dodecyl sulfate, and proteinase inhibitor cocktail (Roche, Mannheim, Germany) with agitation. Subsequently, lysates were cleared by centrifugation at 16000 r.c.f. for $45 \mathrm{~min}$, and protein concentration was measured with BCA assay (Thermo Scientific, Waltham, MA). Lysates containing $200 \mu \mathrm{g}$, and in some experiments $150 \mu \mathrm{g}$ protein, were loaded onto $15 \%$ sodium dodecyl sulfate gel and run for $1 \mathrm{~h}$ at $200 \mathrm{~V}$ and $20 \mathrm{~mA}$ on an EPS-301 electrophoresis power supply (Amersham Biosciences). Proteins were then transferred to a nitrocellulose membrane using Towbin buffer for semi-dry transfer for $32 \mathrm{~min}$ at $25 \mathrm{~V}$ and $0.35 \mathrm{~A}$ using a Trans-blot semi-dry transfer cell system (Bio-Rad, Hercules, CA). Subsequently, the membrane was blocked in $4 \%$ dry milk dissolved in $0.05 \%$ TWEEN/TBS overnight at $4{ }^{\circ} \mathrm{C}$. The membrane was then incubated with $10 \mathrm{ml} 1 \mu \mathrm{g} \mathrm{ml}^{-1}$ mouse anti-human IFN- $\alpha$ antibodies (MabTech, Nacka Strand, Sweden) detecting most subtypes $(1 / 13,2,4,5,6,7,8,10$, 14,16 , and 17), followed by incubation with 1:15000 horseradish peroxidase-labeled goat anti-mouse IgG (Southern Biotech) at RT for an hour. IFN- $\alpha$ was detected with supersignal west pico chemiluminescent substrate from Thermo Scientific. Films were exposed for various periods from 1 to $20 \mathrm{~min}$. Human recombinant IFN- $\alpha 2$ a (Roceron) and a mix of various IFN- $\alpha$ subtypes (positive control for pan-IFN- $\alpha$ ELISA from Mabtech) were used as positive controls.

RT-PCR. Based on HLA-DR and CD45 expression, 20-50,000 cells were sorted from single-cell suspensions derived from duodenal biopsies by digestion. Cells were spun down, resuspended in $200 \mu \mathrm{l}$ RLT lysis buffer, and stored at $-70{ }^{\circ} \mathrm{C}$ until use. Similar number of peripheral blood mononuclear cells that were incubated with either medium or $12.5 \mu \mathrm{g} \mathrm{ml}^{-1} \mathrm{CpG}$ ODN 2216 for $4 \mathrm{~h}$ were used as positive control. RNA was prepared with the microRNAeasy kit (Qiagen, Valencia, CA) according to the manufacturer's instructions, followed by cDNA synthesis. Subsequently, preamplification of all mRNA of interest was performed using Taqman preamp mastermix (Applied Biosystems, Carlsbad, CA) using a 96-well Veriti thermal cycler from Applied Biosystems. In pilot experiments, four housekeeping genes were compared: GAPDH, CKDNB1, CASC3, and RPL13A. The two showing most similar expression across the populations, RPL13A and CASC3, were included in all assays, and RPL13A was used to calculate relative expression. RT-PCR was performed in triplicates in a 384-well $7900 \mathrm{HT}$ fast RT-PCR system (Applied Biosystems). Expression of IFNB1 and five IFN- $\alpha$ subtypes, IFNA1, IFNA2, IFNA4, IFNA5, and IFNA8, were assessed in addition to the expression of IL-28A/IFNL2 and IL-29/IFNL1. All expression assays and master mixes were purchased from Applied Biosystems. 
ELISA. Lysates were prepared from whole biopsies as described in the western blot section, and IFN- $\lambda$ concentration per $50 \mu \mathrm{g}$ protein was measured in duplicates with ELISA using Verikine human interferonlambda kit (PBL Interferonsource, Piscataway, NJ) according to the manufacturer's instructions.

Statistical analysis. Mann-Whitney test was used to assess the difference between densities of PDCs in CD patients and controls. Student's $t$-test was used to compare the relative mRNA expression of IFN- $\alpha$ subtypes in CD patients and controls.

Supplementary Material is linked to the online version of the paper at http://www.nature.com/mi

\section{ACKNOWLEDGEMENTS}

We thank the patients for donating duodenal biopsies. We are grateful to Bjørg Simonsen at the Department of Immunology and to Aaste Aursjø, Hogne Røed Nilsen and Kjersti Thorvaldsen Hagen at the Laboratory for Immunohistochemistry and Immunopathology (Department of Pathology), Oslo University Hospital-Rikshospitalet, for excellent technical assistance.

Funding: This project was supported by grants from the South-Eastern Norway Regional Health Authority.

\section{DISCLOSURE}

The authors declare no conflict of interest.

c) 2013 Society for Mucosal Immunology

\section{REFERENCES}

1. Schulz, O. et al. Intestinal CD103 +, but not CX3CR1 + , antigen sampling cells migrate in lymph and serve classical dendritic cell functions. J. Exp. Med. 206, 3101-3114 (2009).

2. Maloy, K.J. et al. Intestinal homeostasis and its breakdown in inflammatory bowel disease. Nature 474, 298-306 (2011).

3. Varol, C. et al. Intestinal lamina propria dendritic cell subsets have different origin and functions. Immunity 31, 502-512 (2009).

4. Swiecki, M. et al. Unraveling the functions of plasmacytoid dendritic cells during viral infections, autoimmunity, and tolerance. Immunol. Rev. 234, 142-162 (2010).

5. Tezuka, H. et al. Prominent role for plasmacytoid dendritic cells in mucosal T cell-independent IgA induction. Immunity 34, 247-257 (2011).

6. Colonna, M. et al. Plasmacytoid dendritic cells in immunity. Nat. Immunol. 5, 1219-1226 (2004).

7. Fries, P.N. et al. Mucosal dendritic cell diversity in the gastrointestinal tract. Cell Tissue Res. 343, 33-41 (2011).

8. Takenaka, S. et al. Dendritic cells derived from murine colonic mucosa have unique functional and phenotypic characteristics. J. Immunol. 178 7984-7993 (2007).

9. Wendland, M. et al. CCR9 is a homing receptor for plasmacytoid dendritic cells to the small intestine. Proc. Natl. Acad. Sci. USA 104, 6347-6352 (2007).

10. Pabst, O. et al. Cryptopatches and isolated lymphoid follicles: dynamic lymphoid tissues dispensable for the generation of intraepithelial Iymphocytes. Eur. J. Immunol. 35, 98-107 (2005).

11. Hadeiba, $\mathrm{H}$. et al. CCR9 expression defines tolerogenic plasmacytoid dendritic cells able to suppress acute graft-versus-host disease. Nat. Immunol. 9, 1253-1260 (2008).

12. Farkas, L. et al. Plasmacytoid dendritic cells (natural interferon- alpha/betaproducing cells) accumulate in cutaneous lupus erythematosus lesions. Am. J. Pathol. 159, 237-243 (2001).

13. Gregorio, J. et al. Plasmacytoid dendritic cells sense skin injury and promote wound healing through type I interferons. J. Exp. Med. 207, 2921-2930 (2010).

14. Jahnsen, F.L. et al. Experimentally induced recruitment of plasmacytoid (CD123high) dendritic cells in human nasal allergy. J. Immunol. 165 4062-4068 (2000).
15. Jahnsen, F.L. et al. Rapid dendritic cell recruitment to the bronchial mucosa of patients with atopic asthma in response to local allergen challenge. Thorax 56, 823-826 (2001).

16. Nestle, F.O. et al. Plasmacytoid predendritic cells initiate psoriasis through interferon-alpha production. J. Exp. Med. 202, 135-143 (2005).

17. Di Sabatino, A. et al. Evidence for the role of interferon-alfa production by dendritic cells in the Th1 response in celiac disease. Gastroenterology 133 , 1175-1187 (2007)

18. Sollid, L.M. Coeliac disease: dissecting a complex inflammatory disorder. Nat. Rev. Immunol. 2, 647-655 (2002).

19. Monteleone, G. et al. Role of interferon alpha in promoting Thelper cell type 1 responses in the small intestine in coeliac disease. Gut 48, 425-429 (2001).

20. Adinolfi, L.E. et al. Interferon and ribavirin treatment for chronic hepatitis $C$ may activate celiac disease. Am. J. Gastroenterol. 96, 607-608 (2001).

21. Bardella, M.T. et al. Celiac disease during interferon treatment. Ann. Intern. Med. 131, 157-158 (1999).

22. Bourliere, M. et al. Onset of coeliac disease and interferon treatment. Lancet 357, 803-804 (2001).

23. Cammarota, G. et al. Onset of coeliac disease during treatment with interferon for chronic hepatitis C. Lancet 356, 1494-1495 (2000).

24. Lim, E.J. et al. Unmasking of coeliac disease on interferon treatment for hepatitis C. Intern. Med. J. 40, 85-87 (2010).

25. Martins, Jr E.V. et al. Celiac disease onset after pegylated interferon and ribavirin treatment of chronic hepatitis C. Arq. Gastroenterol. 41, 132-133 (2004)

26. Raki, M. etal. A unique dendritic cell subset accumulates in the celiac lesion and efficiently activates gluten-reactive T cells. Gastroenterology 131, 428-438 (2006).

27. Beitnes, A.C. et al. Density of CD163(+) CD11C $(+)$ dendritic cells increases and CD103(+) dendritic cells decreases in the coeliac lesion. Scand. J. Immunol. 74, 186-194 (2011).

28. Pott, J. et al. IFN-lambda determines the intestinal epithelial antiviral host defense. Proc. Natl. Acad. Sci. USA 108, 7944-7949 (2011).

29. Stene, L.C. et al. Rotavirus infection frequency and risk of celiac disease autoimmunity in early childhood: a longitudinal study. Am. J. Gastroenterol. 101, 2333-2340 (2006).

30. Dzionek, A. et al. BDCA-2, BDCA-3, and BDCA-4: three markers for distinct subsets of dendritic cells in human peripheral blood. J. Immunol. 165, 6037-6046 (2000).

31. van der Sluijs, K.F. et al. Monocyte Differentiation Towards Regulatory Dendritic Cells is not Affected by RSV-induced Inflammatory Mediators. Am. J. Respir. Cell Mol. Biol. 44, 655-664 (2010).

32. Dillon, S.M. et al. Human intestinal lamina propria CD1c + dendritic cells display an activated phenotype at steady state and produce IL-23 in response to TLR7/8 stimulation. J. Immunol. 184, 6612-6621 (2010).

33. Benahmed, M. et al. Inhibition of TGF-beta signaling by IL-15: a new role for $\mathrm{IL}-15$ in the loss of immune homeostasis in celiac disease. Gastroenterology 132, 994-1008 (2007).

34. Pizarro, T.T. et al. IL-18, a novel immunoregulatory cytokine, is up-regulated in Crohn's disease: expression and localization in intestinal mucosal cells. J. Immunol. 162, 6829-6835 (1999).

35. Moll, H.P. et al. The differential activity of interferon-alpha subtypes is consistent among distinct target genes and cell types. Cytokine 53, 52-59 (2011)

36. Siegal, F.P. et al. The nature of the principal type 1 interferon-producing cells in human blood. Science 284, 1835-1837 (1999).

37. Larrea, E. et al. IFN-alpha5 mediates stronger Tyk2-stat-dependent activation and higher expression of 2',5'-oligoadenylate synthetase than IFN-alpha2 in liver cells. J. Interferon Cytokine Res. 24, 497-503 (2004).

38. Antonelli, A. et al. Hepatitis C: thyroid dysfunction in patients with hepatitis C on IFN-alpha therapy. Nat. Rev. Gastroenterol. Hepatol. 6, 633-635 (2009).

39. Haller, O. et al. Interferon-induced Mx proteins in antiviral host defense. Biochimie 89, 812-818 (2007).

40. Flohr, F. et al. The central interactive region of human MxA GTPase is involved in GTPase activation and interaction with viral target structures. FEBS Lett. 463, 24-28 (1999). 